Article

\title{
Ceramic Identity Contributes to Mechanical Properties and Osteoblast Behavior on Macroporous Composite Scaffolds
}

\author{
Diana G. Morales-Hernandez ${ }^{1}$, Damian C. Genetos ${ }^{2}$, David M. Working ${ }^{1}$, Kaitlin C. Murphy ${ }^{1}$ \\ and J. Kent Leach ${ }^{1,3, *}$
}

1 Department of Biomedical Engineering, University of California, Davis, CA 95616, USA;

E-Mails:dgmorales@ucdavis.edu (D.G.M.); workingdm@gmail.com (D.M.W.);

kmurphy@ucdavis.edu (K.C.M.)

2 Department of Anatomy, Physiology, and Cell Biology, School of Veterinary Medicine, University of California, Davis, CA 95616, USA; E-Mail: dgenetos@ucdavis.edu

3 Department of Orthopaedic Surgery, School of Medicine, University of California, Davis, Sacramento, CA 95817, USA

* Author to whom correspondence should be addressed; E-Mail: jkleach@ucdavis.edu; Tel.: +1-530-754-9149; Fax: +1-530-754-5739.

Received: 16 March 2012; in revised form: 27 April 2012 / Accepted: 17 May 2012 /

Published: 23 May 2012

\begin{abstract}
Implants formed of metals, bioceramics, or polymers may provide an alternative to autografts for treating large bone defects. However, limitations to each material motivate the examination of composites to capitalize on the beneficial aspects of individual components and to address the need for conferring bioactive behavior to the polymer matrix. We hypothesized that the inclusion of different bioceramics in a ceramic-polymer composite would alter the physical properties of the implant and the cellular osteogenic response. To test this, composite scaffolds formed from poly(lactide-co-glycolide) (PLG) and either hydroxyapatite (HA), $\beta$-tricalcium phosphate (TCP), or bioactive glass (Bioglass $\left.45 \mathrm{~S}^{\circledR}, \mathrm{BG}\right)$ were fabricated, and the physical properties of each scaffold were examined. We quantified cell proliferation by DNA content, osteogenic response of human osteoblasts (NHOsts) to composite scaffolds by alkaline phosphatase (ALP) activity, and changes in gene expression by qPCR. Compared to BG-PLG scaffolds, HA-PLG and TCP-PLG composite scaffolds possessed greater compressive moduli. NHOsts on BG-PLG substrates exhibited higher ALP activity than those on control, HA-, or TCP-PLG scaffolds after 21 days, and cells on composites exhibited a 3-fold increase in ALP activity between
\end{abstract}


7 and 21 days versus a minimal increase on control scaffolds. Compared to cells on PLG controls, RUNX2 expression in NHOsts on composite scaffolds was lower at both 7 and 21 days, while expression of genes encoding for bone matrix proteins (COL1A1 and $S P A R C)$ was higher on BG-PLG scaffolds at both time points. These data demonstrate the importance of selecting a ceramic when fabricating composites applied for bone healing.

Keywords: bioceramic; hydroxyapatite; bioactive glass; composite; scaffold; bone

\section{Introduction}

The treatment of slow or nonhealing bone fractures is a significant clinical problem. Implants formed of metals, bioceramics, polymers, and decellularized tissues are under investigation to reduce or eliminate the current limitations of the "gold standard" of autograft bone. However, each of these materials also presents challenges in their application including wear debris formation and stress shielding, inadequate porosity to allow cellular infiltration, inability to be resorbed, or undesirable inflammatory responses [1]. Moreover, with the exception of polymeric systems, significant challenges exist to tailor these implants for the specific defect requiring treatment.

Calcium phosphate ceramics and bioactive glasses share similarities between their surface composition and chemical structure and the mineral phase of bone, and demonstrate enhanced osteoconductivity under in vivo settings [2]. Hydroxyapatite $\left(\mathrm{HA} ; \mathrm{Ca}_{10}\left(\mathrm{PO}_{4}\right)_{6}(\mathrm{OH})_{2}\right)$ is the major mineral component of bone and is widely used as a bone substitute, both as a homogeneous implant and as a component of composite materials [3]. Beta-tricalcium phosphate $\left(\beta-\mathrm{TCP} ; \mathrm{Ca}_{3}\left(\mathrm{PO}_{4}\right)_{2}\right)$ shares similar chemical composition as $\mathrm{HA}$, but resorbs faster due to its lower $\mathrm{Ca} / \mathrm{P}$ ratio and is weaker than HA per unit mass, making its use in treating defects in load-bearing bones more challenging [4]. Bioactive glasses (BG) exhibit tissue stimulatory properties and are under extensive investigation for their potential use in engineering of hard tissues [5,6]. However, the formation of implants which are wholly composed of bioceramics requires high temperatures, controlled cooling, and the resulting materials are brittle and slowly resorbable [7].

The development of matrices that possess sufficient strength, osteoconductivity, porosity, and degradation times represents a major focus in the arena of bone repair. Polymers derived from synthetic materials are commonly biocompatible, bioresorbable, and tailorable materials that can be molded into highly porous scaffolds [8]. For example, poly(lactide-co-glycolide) (PLG) is a commonly-used polymer for bridging bone tissue defects due to the ease of tailoring the degradation time by modulating the ratio of lactide and glycolide monomers during synthesis $[9,10]$. However, scaffolds formed of PLG lack sufficient mechanical strength for withstanding load, desirable osteoconductivity for integration with surrounding bone, or fail to provide instructional cues to the resident cells [11]. Moreover, there is a pressing need for conferring bioactive behavior to the polymer matrix.

In response to limitations of implants formed solely of bioceramics and polymers, we and others have developed composite scaffolds to capitalize on the beneficial aspects of the individual components. Polymers reinforced with bioceramics, fabricated using a variety of methods, consistently 
demonstrate improved mechanical properties over polymeric substrates without the constraints required of producing 3D bioceramic implants. The osteoconductivity and osteogenic potential of polymers is increased upon the addition of ceramics such as bone-like mineral, HA, TCP, and BG [12-16]. Composite scaffolds can have profound effects on other aspects of bone repair beyond inducing osteogenic differentiation. For example, we demonstrated that scaffolds containing nanosized HA enhance the osteogenic response and upregulate the secretion of potent proangiogenic trophic factors from human mesenchymal stem cells, thereby increasing the persistence of implanted cells, accelerating neovascularization, and enhancing bone formation [17,18]. BG stimulates angiogenesis in vivo [6,19], and vessel density and the quality of new bone formation was increased in calvarial defects treated with BG-coated scaffolds [20].

Despite significant evidence demonstrating the efficacy of bioceramic-polymer composite scaffolds for bone formation, little is known regarding potential differences in osteogenesis using composite scaffolds containing differing bioceramic particulates. We hypothesized that the identity of the ceramic incorporated within macroporous polymer scaffold composites would contribute to its material properties and osteogenic potential. In this study, composite scaffolds were fabricated using three common calcium phosphate materials: HA, TCP, or BG. We report that the addition of any bioceramic increases scaffold stiffness, decreases porosity, and differentially directs osteogenesis of NHOsts, with BG-loaded scaffolds potently stimulating osteogenesis compared to scaffolds containing other bioceramics.

\section{Experimental Section}

\subsection{Scaffold Preparation}

Scaffolds were prepared using a gas foaming/particulate leaching method as described [18,21]. Briefly, PLG microspheres (85:15 DLG 7E; Lakeshore Biomaterials, Birmingham, AL, USA) were prepared using a double emulsion process. Bioceramic particulate, lyophilized microspheres, and $\mathrm{NaCl}$ particles (250-425 $\mu \mathrm{m}$ in diameter) were mixed in a 2.5:1:19 ratio, while control scaffolds were prepared without bioceramic. The selection of mass ratio was derived from our previous studies demonstrating increased stiffness while maintaining porosity in macroporous scaffolds at this mass ratio [18]. Composite scaffolds were fabricated using hydroxyapatite (HA, $100 \mathrm{~nm}$ diameter; Berkeley Advanced Biomaterials, Berkeley, CA, USA), $\beta$-tricalcium phosphate (TCP, $<200 \mathrm{~nm}$ particle size, Sigma Aldrich, St. Louis, MO, USA), or 45S5 Bioglass ${ }^{\circledR}$ (BG, 90-170 $\mu \mathrm{m}$ particle size, Novabone, Alachua, FL, USA). Mixtures were compressed in a stainless steel die using a Carver Press (Fred S. Carver) at $10 \mathrm{MPa}$ for $1 \mathrm{~min}$ to produce solid disks ( $8.5 \mathrm{~mm}$ diameter, $1.5 \mathrm{~mm}$ thick). Disks were then placed under high pressure $\mathrm{CO}_{2}$ gas $(5.5 \mathrm{MPa})$ for $16 \mathrm{~h}$, after which the pressure was rapidly released to ambient to achieve polymer fusion. Solid disks were leached in distilled water for $24 \mathrm{~h}$ to remove the $\mathrm{NaCl}$ particles and generate highly porous scaffolds.

\subsection{Scaffold Characterization}

Gross morphology of scaffolds was determined by scanning electron microscope images. Scaffolds were gold-coated using a sputter coater (Desk II; Denton Vacuum, Moorestown, NJ, USA). Specimens were imaged with a Hitachi S3500-N Scanning Electron Microscope at $10 \mathrm{kV}$. Pore diameter was 
quantified from scanning electron microscope images by measuring the long-axis of 20-40 pores in each scaffold using NIH Image J. Scaffold porosity was determined using Archimedes' method [18]. Scaffolds were submerged in $100 \% \mathrm{EtOH}$ in a custom-made vacuum bottle for 5 min until all bubbles were removed from the pores. The weight of the scaffolds before and after immersion was recorded, and scaffold porosity was calculated.

The distribution of bioceramic particulate throughout the scaffolds was grossly observed by adsorption of Trypan blue as described [18,22]. Scaffolds were exposed to a $0.4 \%(\mathrm{w} / \mathrm{v})$ Trypan blue solution (Alfa Aesar, Ward Hill, MA, USA) for $10 \mathrm{~s}$. Scaffolds were rinsed twice in distilled $\mathrm{H}_{2} \mathrm{O}$ and placed in $100 \% \mathrm{EtOH}$ for $1 \mathrm{~min}$. Scaffolds were sonicated for $5 \mathrm{~s}$ at $40 \%$ power in $100 \% \mathrm{EtOH}$ to remove remaining unbound dye and rinsed in distilled $\mathrm{H}_{2} \mathrm{O}$ before drying and analysis. The efficiency of bioceramic incorporation was determined by measuring the mass of individual components and final scaffold mass after fabrication.

Scaffold stiffness was determined by measuring the compressive modulus using an Instron 5,800 Series Testing System (Norwood, CA, USA). Samples were compressed with a constant deformation rate of $1 \mathrm{~mm} / \mathrm{min}$. The compressive modulus was calculated from the first $5 \%$ of the strain [18].

\subsection{Cell Culture}

Normal human osteoblasts (NHOsts) were purchased from Lonza (Clonetics ${ }^{\circledR}$, Walkersville, MD, USA). NHOsts were expanded in DMEM (Invitrogen, Carlsbad, CA, USA) containing 10\% fetal bovine serum (FBS, JR Scientific, Woodland, CA, USA) and 1\% penicillin/streptomycin (Mediatech, Manassas, VA, USA) in standard cell culture conditions $\left(37^{\circ} \mathrm{C}, 5 \% \mathrm{CO}_{2}, 21 \% \mathrm{O}_{2}\right)$. Experiments were performed with cells between passages $2-5$.

\subsection{Osteogenic Potential}

Scaffolds were sterilized in $95 \% \mathrm{EtOH}$ for $30 \mathrm{~min}$, rinsed twice with sterile PBS over $30 \mathrm{~min}$, and incubated in DMEM for $30 \mathrm{~min}$ prior to cell seeding. NHOsts were statically seeded at $7.5 \times 10^{6}$ cells $/ \mathrm{cm}^{3}$ and allowed to attach for $1 \mathrm{~h}$ before moving cell-seeded constructs into DMEM. On day 0 (24 h post cell seeding), media was exchanged for fresh DMEM containing osteogenic supplements composed of $10 \mathrm{mM} \beta$-glycerophosphate and $50 \mu \mathrm{g} / \mathrm{mL}$ ascorbate-2-phosphate. Scaffolds were maintained under standard cell culture conditions on an XYZ shaker to enhance transport within the 3D construct, and media was changed every 3-4 days.

Samples were collected and analyzed after $4 \mathrm{~h}$ to assess cell seeding efficiency, or after 7 or 21 days to determine osteogenic potential. Briefly, scaffolds were rinsed in PBS and minced with a razor blade, incubated in 1X passive lysis buffer (Promega, San Luis Obispo, CA, USA) at room temperature for $10 \mathrm{~min}$, sonicated briefly, and centrifuged at 10,000 rpm for $5 \mathrm{~min}$. The supernatant was assayed for alkaline phosphatase (ALP) activity by incubating with $50 \mathrm{mM}$-nitrophenyl phosphate (PNPP) in an assay buffer $\left(100 \mathrm{mM}\right.$ glycine, $\left.1 \mathrm{mM} \mathrm{MgCl}_{2}, \mathrm{pH}=10.5\right)$ at $37{ }^{\circ} \mathrm{C}$ [12,23]. Absorbance was measured at $405 \mathrm{~nm}$ and converted to ALP activity using the extinction coefficient for PNPP $\left(1.85 \times 10^{4} \mathrm{M}^{-1} \cdot \mathrm{cm}^{-1}\right)$. DNA content was quantified from lysate using a Quant-iT PicoGreen dsDNA kit (Invitrogen). To determine cell distribution, scaffolds were seeded with NHOsts and cultured for 
1 day, decalcified in Calci-Clear (National Diagnostics, Atlanta, GA, USA) for 3 days, and hemotoxylin and eosin staining was performed on paraffin-embedded sections at $5 \mu \mathrm{m}$ thickness.

The expression of genes associated with osteogenesis was measured in NHOsts seeded on composite scaffolds using qPCR. Briefly, total RNA from scaffolds was collected using an RNeasy Mini kit (Qiagen, Valencia, CA, USA) at 7 and 21 days. Between 200-1,000 ng of total RNA was reverse-transcribed with Superscript II Reverse Transcriptase (Invitrogen). Quantitative PCR was performed using primers and probes for RUNX2, COL1A1, and SPARC (Applied Biosystems, Foster City, CA, USA) on a Mastercycler ${ }^{\circledR}$ realplex2 (Eppendorf, Westbury, NY, USA). Amplification conditions were $50{ }^{\circ} \mathrm{C}$ for $2 \mathrm{~min}, 95^{\circ} \mathrm{C}$ for $10 \mathrm{~min}$, followed by 40 cycles at $95^{\circ} \mathrm{C}$ for $15 \mathrm{~s}$ and $60^{\circ} \mathrm{C}$ for 1 min. Quantitative PCR results were normalized to RPL13 transcript level to yield $\Delta \mathrm{C}_{\mathrm{t}}$. Fold change in expression was subsequently calculated using the formula $2^{-\Delta \mathrm{Ct}}$ [24].

\subsection{Statistical Analysis}

Results are expressed as mean \pm standard deviation (SD) of the mean, assuming normal distribution of data sets, with the exception of PCR data, which is expressed as mean \pm standard error of the mean (SEM). Statistical analyses were performed between two groups using the Student's t-test or between multiple groups using a one-way ANOVA with Student Newman-Keuls multiple comparison post hoc test in GraphPad Prism ${ }^{\circledR} 5$ analysis software (GraphPad Software, San Diego, CA, USA). Probability values $(p)$ for significance were calculated; $p<0.05$ was considered statistically significant.

\section{Results and Discussion}

\subsection{Scaffold Characterization}

Bioceramic incorporation in macroporous scaffolds was highly efficient, as we did not detect any measurable loss in weight after scaffold fabrication for any group. We observed similar pore diameters for each scaffold type when imaging cross-sections using scanning electron microscopy (Figure 1a). The edges of the pores appeared rougher and less defined in composite scaffolds compared to PLG control scaffolds. We observed homogenous distribution of bioceramic particulate throughout the scaffolds when qualitatively assessed by Trypan blue adsorption (Figure 1b). HA-containing scaffolds adsorbed more dye than other composites. Scaffolds stained uniformly, despite differences in intensity for the stain, thus confirming homogenous distribution of the bioceramic throughout the composite scaffold. Composite scaffolds exhibited significant reductions in porosity compared to control scaffolds $(93.0 \pm 1.7 \%$; Figure 1c). However, composites formed with HA and TCP had similar porosities $(82.4 \pm 1.3 \%$ and $80.8 \pm 5.3 \%$ ), respectively, while scaffolds containing BG showed reduced porosity $(64.2 \pm 6.8 \%)$. Moreover, scaffolds formed with BG exhibited lower pore diameters compared to other substrates (Figure 1d), suggesting that the ceramic was not entirely embedded within the polymer. 
Figure 1. (a) Construct morphology was observed using scanning electron microscopy. Substrates imaged at 100X; scale bar represents $200 \mu \mathrm{m}$; (b) The presence and distribution of bioceramic was qualitatively observed by Trypan blue staining; (c) Scaffold porosity was determined using Archimedes' method; (d) Scaffold pore diameter from each scaffold formulation. Data are mean $\pm \mathrm{SD}(\mathrm{n}=5$ for $\mathbf{a}-\mathbf{c} ; \mathrm{n}=20-40$ for $(\mathbf{d}))$. ${ }^{* * *} p<0.0001 v s$. PLG; $* * p<0.001$ vs. PLG; $\$ p<0.05$ vs. PLG; \# $p<0.0001$ vs. HA-PLG and TCP-PLG; $\% p<0.001 v s$. TCP-PLG.

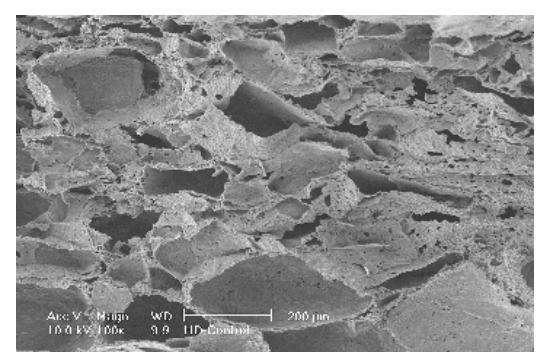

PLG

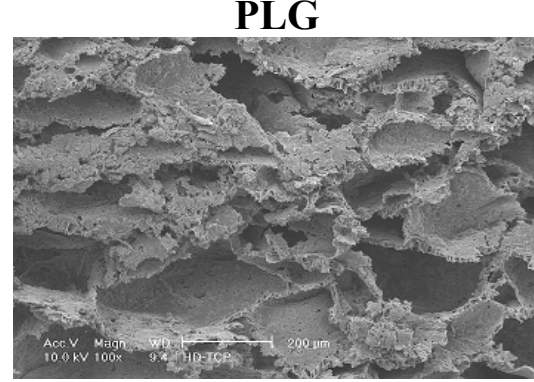

TCP-PLG

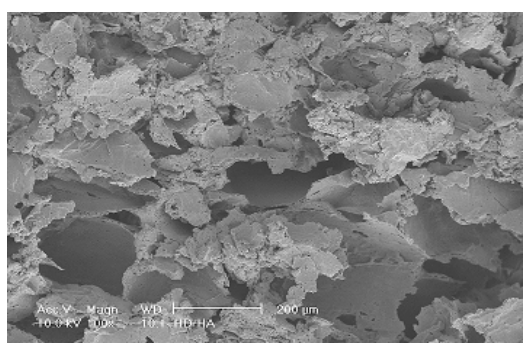

HA-PLG

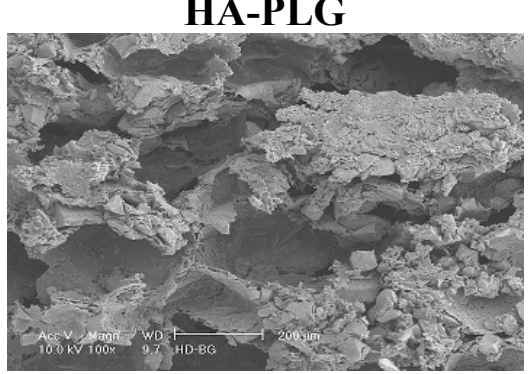

BG-PLG

(a)

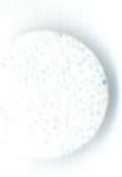

PLG

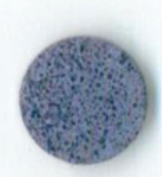

HA-PLG
TCP-PLG

(b)

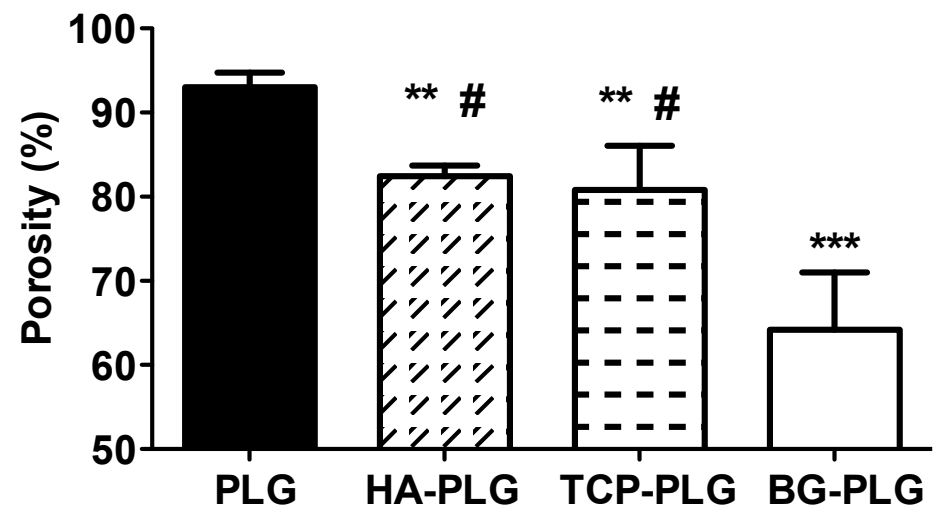

(c) 
Figure 1. Cont.

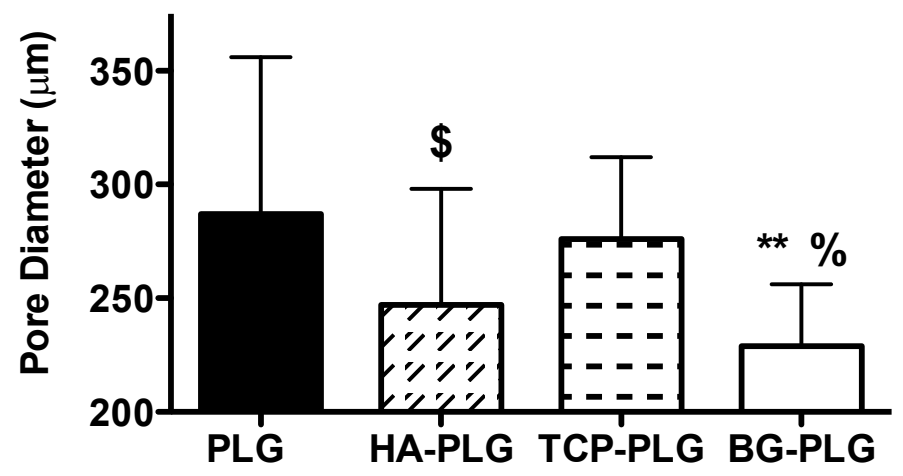

(d)

Compressive moduli increased upon the addition of any bioceramic. Composite scaffolds containing HA and TCP exhibited a 3-4 fold increase in compressive modulus compared to PLG scaffolds, while substrates containing BG exhibited a 2.5 fold greater compressive modulus, on average, versus control scaffolds (Figure 2).

Figure 2. Influence of bioceramic on mechanical properties of substrate. Compressive modulus increased with the addition of bioceramic. Data are mean $\pm \mathrm{SD}(\mathrm{n}=9$ for PLG scaffolds, $\mathrm{n}=5$ for composite scaffolds). ${ }^{* * *} p<0.0001$ vs. PLG; $* * p<0.001$ vs. PLG; $\# p<0.05$ vs. HA-PLG and TCP-PLG.

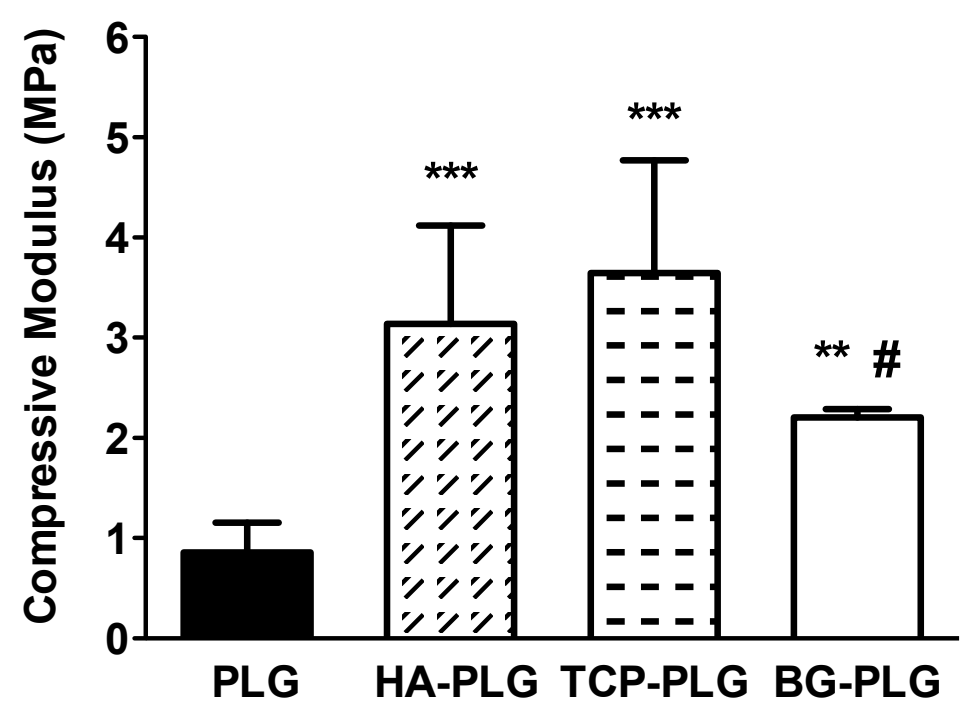

\subsection{Osteoconductive Potential of Composite Scaffolds}

Upon examination by hemotoxylin and eosin staining, cells appeared to adhere predominantly to the outer surface of the scaffold (Figure 3). There were no statistically significant differences in cell seeding efficiency (Figure 4a). The ability of each scaffold to support cell proliferation and survival was assessed by quantifying DNA content on each scaffold after 7 or 21 days in culture. After 21 days, DNA content per scaffold was reduced in scaffolds containing PLG, HA, or TCP (Figure 4b). 
Figure 3. Distribution of cells on scaffolds observed by H\&E staining. Images at $200 \times$ magnification (scale bar represents $100 \mu \mathrm{m}$ ).

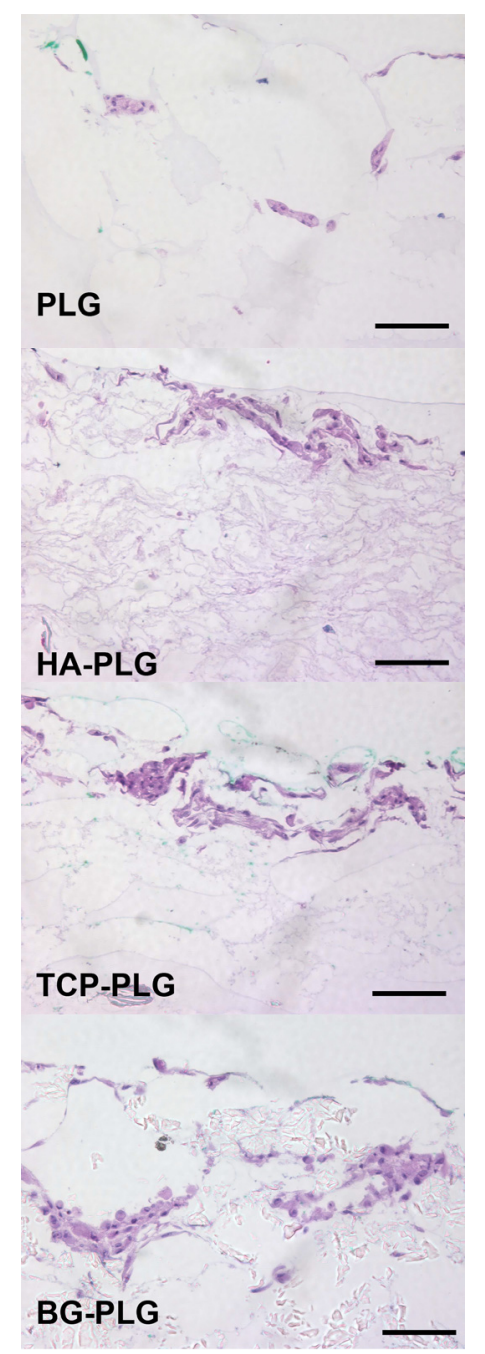

Figure 4. NHOst seeding efficiency (a) and proliferation (b) was measured by quantifying total DNA mass on 3D scaffolds. Data are mean $\pm \mathrm{SD}(\mathrm{n}=4) .{ }^{*} p<0.05$ vs. PLG control; $* * * p<0.001 v s$. PLG; $\dagger \uparrow p<0.001$ vs. BG-PLG; \# $p<0.05$ vs. TCP-PLG.

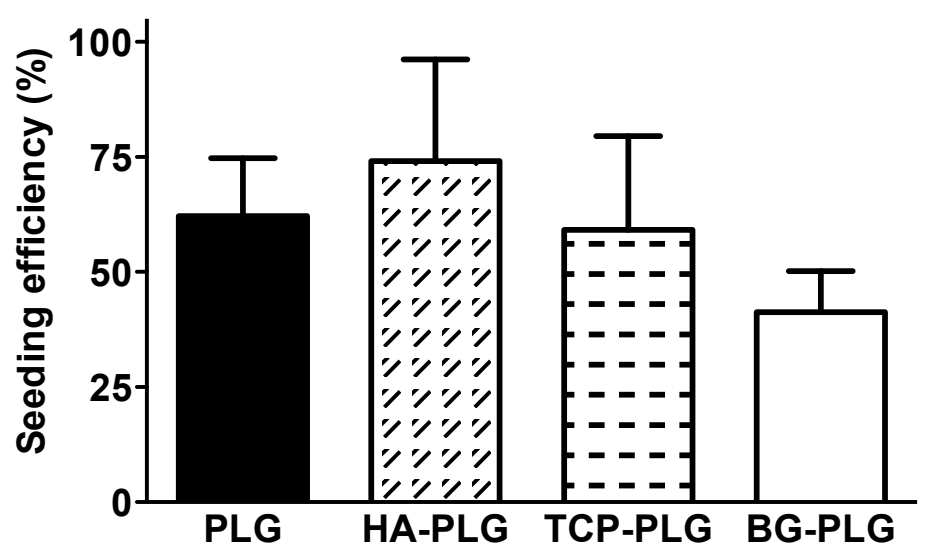

(a) 
Figure 4. Cont.

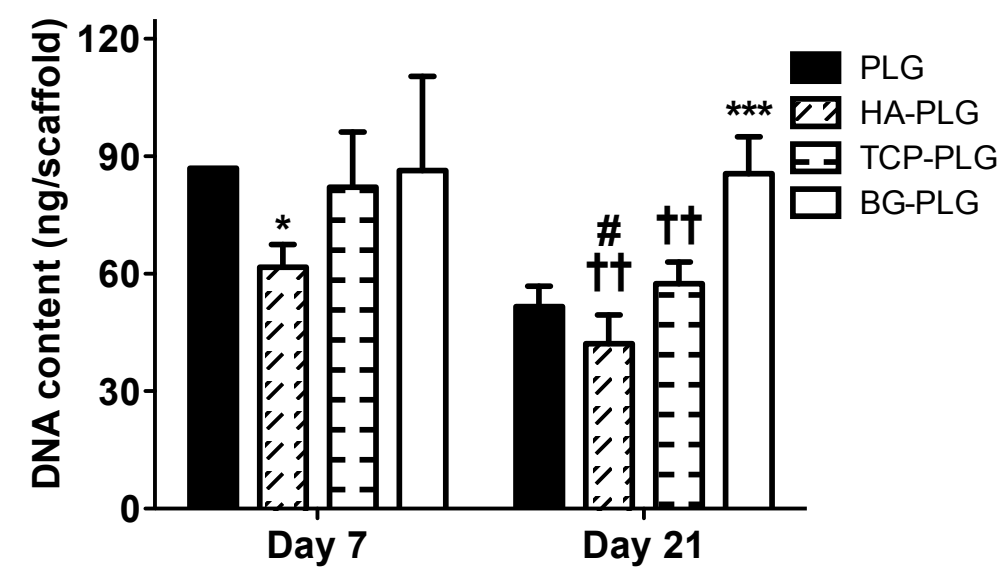

(b)

\subsection{Osteogenic Response of NHOsts}

Changes in ALP activity were monitored as an indicator of osteoblastic differentiation of NHOsts as a function of scaffold composition (Figure 5). At Day 7, cells on HA-containing scaffolds exhibited significantly decreased ALP activity compared to PLG scaffolds, while cells on PLG, TCP-PLG, and BG-PLG exhibited statistically similar levels of enzymatic activity. After 3 weeks of culture, NHOsts on BG-PLG demonstrated significantly increased ALP activity compared to all other groups, while the remaining groups induced similar ALP activity. ALP activity increased over the 3-week culture period for all scaffolds. However, cells on composite scaffolds exhibited a comparable 3-4-fold increase in enzyme activity, while cells on control scaffolds demonstrated only a marginal increase over 21 days.

Figure 5. Alkaline phosphatase activity for NHOsts cultured on macroporous scaffolds. Data are mean $\pm \mathrm{SD}(\mathrm{n}=4) . * p<0.05 v s$. PLG at Day $7 .{ }^{*} p<0.01 v s$. PLG, HA-PLG, and TCP-PLG at Day 21.

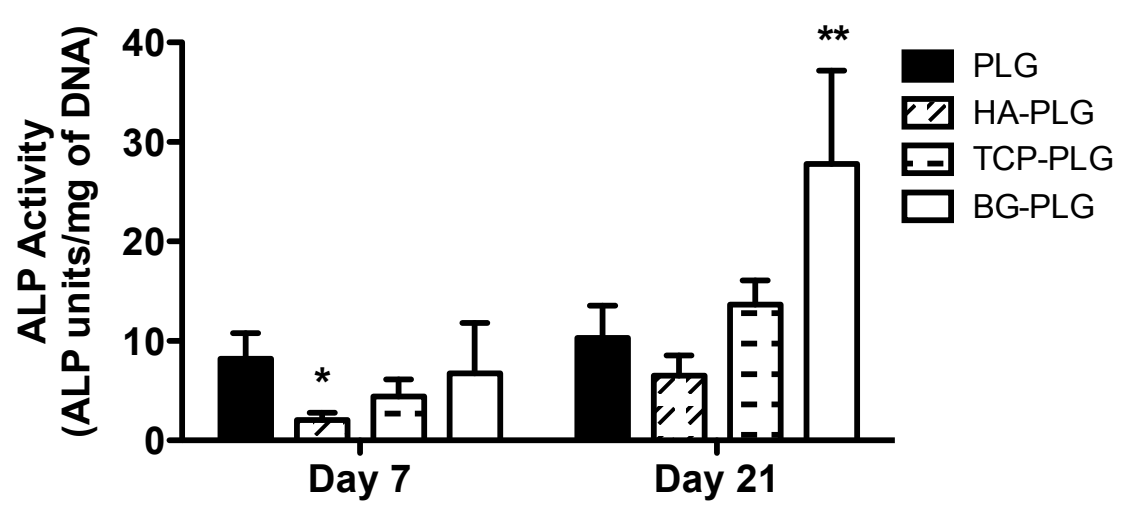

The expression of osteogenic marker genes $R U N X 2, C O L 1 A 1$, and SPARC was analyzed by qPCR from 3D cultures after 7 or 21 days of culture. $R U N X 2$ is an obligate transcription factor for, and early indicator of, osteogenesis [25,26]. NHOsts on PLG control scaffolds exhibited greater RUNX2 expression at both time points compared to cells on any composite scaffold (Figure 6a). Cells on HA- and TCP-containing scaffolds demonstrated significantly lower RUNX2 expression than cells on 
control scaffolds at both time points. RUNX2 expression of cells seeded on HA-PLG scaffolds was significantly lower than cells on BG composite scaffolds at Day 7, while cells on TCP scaffolds expressed lower RUNX2 levels than those on BG composite scaffolds at Day 21.

Figure 6. Quantitative PCR results for genes monitored in NHOsts over 3 weeks cultured on macroporous scaffolds: RUNX2 (a), COL1A1 (b), and SPARC (c). Values reflect fold change in the target mRNA expression over RPL13 vs. Day 7 PLG. Data are mean \pm SEM $(\mathrm{n}=4) .{ }^{*} p<0.05$ vs. PLG control; ** $p<0.01$ vs. PLG; *** $p<0.001$ vs. PLG; $\# p<0.05$ vs. HA-PLG; \#\# $p<0.001$ vs. HA-PLG; $\$ p<0.001$ vs. TCP-PLG; $\dagger p<0.05$ vs. BG-PLG; $\dagger \dagger p<0.001 v s$. BG-PLG.

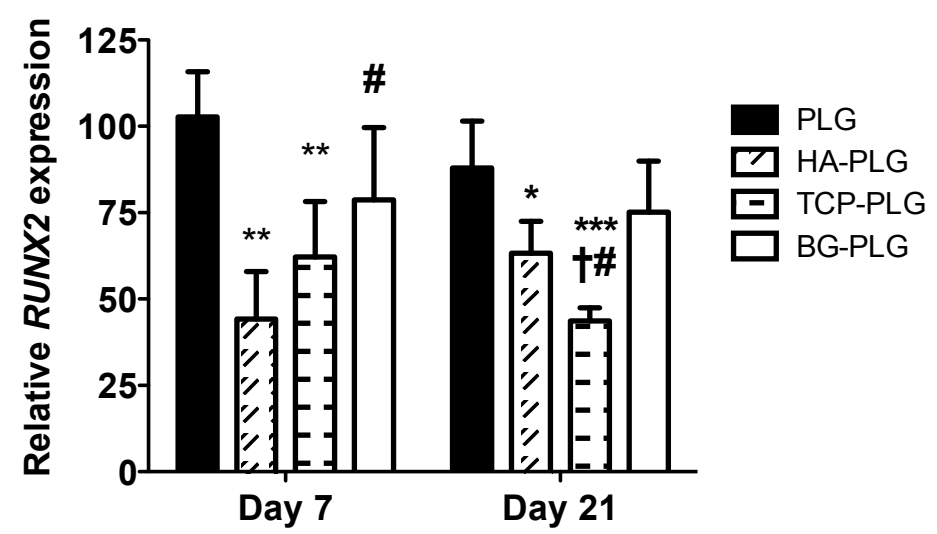

(a)

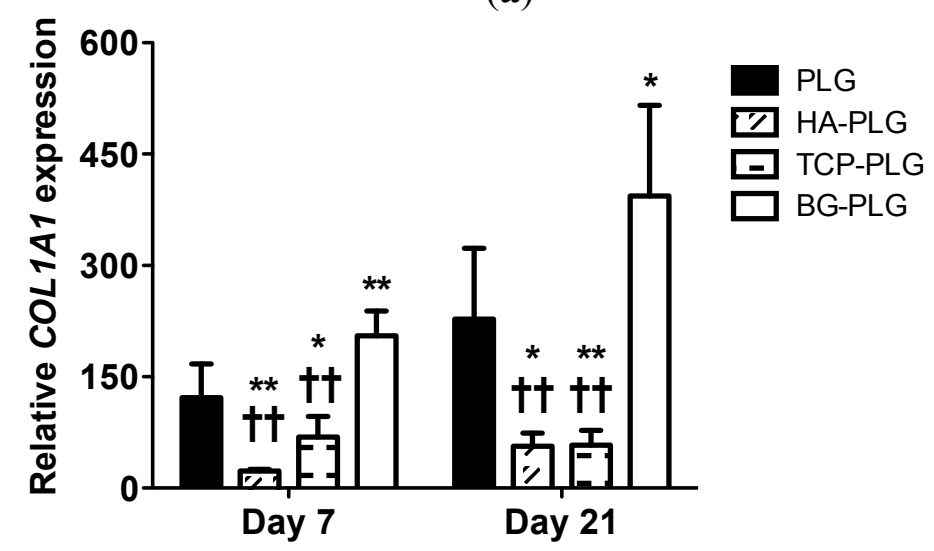

(b)

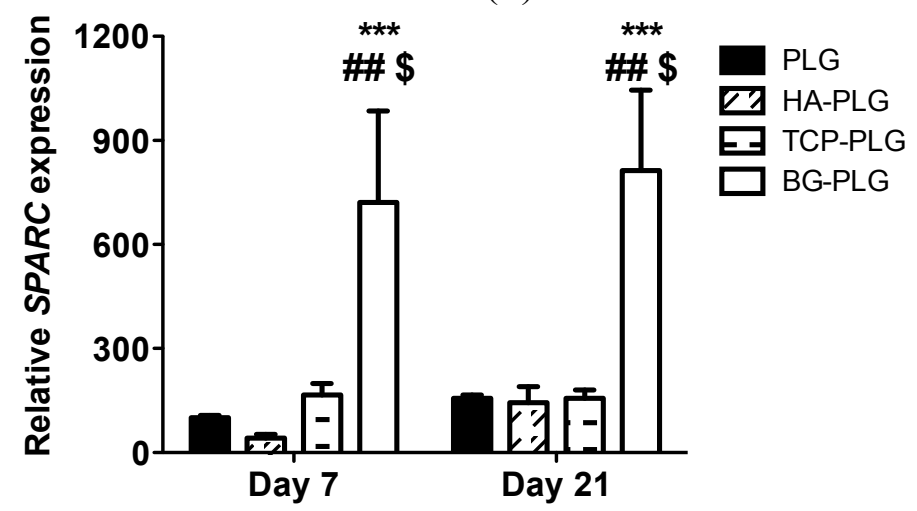

(c) 
Type I collagen is a major constituent of the organic matrix of bone, and COL1A1 encodes for two of the three fibrils that compose collagen I filaments [27]. The influence of substrate composition on COL1A1 transcript exhibited similar trends to RUNX2 expression (Figure 6b). Specifically, cells on BG-PLG scaffolds demonstrated greater COL1A1 expression versus all other composite scaffolds at Day 7, with cells on HA- and TCP-loaded scaffolds possessing significantly lower expression compared to PLG controls. After 21 days, NHOsts on BG-PLG scaffolds expressed significantly greater COL1A1 transcript compared to the other composite substrates, and cells on HA- and TCP-containing scaffolds behaved similar to that of 7 days.

Osteonectin, encoded by the gene $S P A R C$, is a bone-specific protein that is selectively bound to insolubilized type I collagen to enhance the complex binding of synthetic apatite crystals and free calcium ions, thus promoting the nucleation of mineral [28]. Thus, SPARC is a later marker of osteogenesis and signifies the onset of mature bone formation. SPARC gene expression profiles were similar to COL1A1 and remained relatively constant in all groups throughout the study period (Figure 6c). NHOsts on BG-PLG scaffolds exhibited significantly higher SPARC expression at both 7 and 21 days, more than 4.3- and 5.2-fold, respectively, compared to remaining scaffolds.

We sought to explore the contribution of individual bioceramics present within composite implants that are designed for applications in bone tissue engineering. Specifically, we aimed to determine if different ceramics would induce differences in physical properties when fabricating macroporous composite scaffolds, as well as the resulting osteogenic response of human osteoblasts. These data confirm that the resultant physical properties of composite scaffolds are dependent upon ceramic identity, while materials incorporated within osteoconductive bioceramic composite scaffolds differentially direct the behavior of normal human osteoblasts.

Scaffolds that are designed for bridging bone defects and serving as successful cell carriers should ideally be highly porous in order to facilitate cellular invasion and host neovascularization, enable efficient transport of nutrients and waste removal to support cell survival, and promote integration with surrounding bone. The composition of such composite scaffolds is a critical mediator for material properties and cellular response. We fabricated scaffolds with a constant 2.5:1 mass ratio of ceramic to polymer based on our previously published studies that confirmed robust osteogenic response of human mesenchymal stem cells while maintaining high scaffold porosity [18]. Moreover, the compressive moduli of all composite scaffolds were within the lower limit of the compressive strength of trabecular bone (2-12 $\mathrm{MPa}$ ) [29], while producing a scaffold with pore diameters large enough to enable vascularization and bone ingrowth [30]. By fixing the mass ratio of polymer, bioceramic, and porogen during the fabrication process, we discovered that the bioceramic identity strongly contributes to the resulting pore structure and porosity, likely due to interactions between the ceramic and polymer during the gas foaming/particulate leaching process. Pores of composite scaffolds exhibited a more irregular geometry than pores within control scaffolds. PLG control scaffolds were more than $90 \%$ porous, while the addition of HA and TCP reduced composite scaffold porosity to approximately $80 \%$. The addition of BG further reduced scaffold porosity without visible differences in pore structure, suggesting that BG may occlude the micropores within the substrate, thus potentially limiting the success of this implant from the standpoint of diffusional transport to entrapped cells. Indeed, composite scaffolds containing BG possessed the smallest pore diameter, thus supporting the hypothesis that the ceramic is not fully embedded in the polymer. The decreased porosity of BG-PLG 
scaffolds may relate to the significantly increased particulate diameters used compared to HA and TCP. However, we aimed to fabricate composite scaffolds from commercially available bioceramics. Thus, the effect of nanosized BG incorporated into composite scaffolds on osteogenic response justifies further examination.

While scaffold porosity is an important factor relating to vascularization and nutrient transport, other factors may contribute to the success of the implant and the cellular response including substrate stiffness and exposure of ceramic from polymer. These two phenomena are likely linked due to the inherent hydrophilicity of most ceramic materials and hydrophobicity of most polymers. The incorporation of bioceramics into polymer composites increased the compressive strength of all materials. Although increases in substrate stiffness are commonly reported with ceramic-polymer composites, the relatively small increase in compressive modulus may be attributed to the lack of interfacial bonding strength between the ceramic phase and the polymer matrix [31]. Additionally, increases in substrate stiffness are likely due to a reduction in void space within the construct, as well as the ability of embedded material to support compressive load. In agreement with previous studies from our laboratory and others [14,18,32], we observed a corresponding increase in compressive modulus with decreasing porosity for both HA- and TCP-loaded scaffolds. However, BG-PLG scaffolds exhibited a lower compressive modulus than the other composite scaffolds while producing an even lower porosity. These data suggest that BG is not embedded within the polymer during gas foaming as effectively as the other bioceramics, and this may enhance osteogenesis by increasing the availability of osteostimulative ions resulting from BG dissolution to surrounding cells [33].

These data suggest that other factors beyond bulk mechanical properties may contribute to the cellular response. The exposure of ceramic resulting from differences in partitioning from the polymer, as well as ionic dissolution from the ceramic filler, may have a profound effect on the osteogenic potential of associated cells. Composites containing HA, which were previously coated with sucrose to minimize embedding in the polymer and thus maximize access and availability to surrounding cells, demonstrated significant increases in bone formation compared to scaffolds without sucrose-coated HA [34]. These data suggest that ceramic exposure is an important aspect to consider when designing these materials. Strategies to control interfacial bonding strength, perhaps by alkaline treatment to increase roughness and surface area [35], merit further investigation. Previous studies have reported the importance of the ionic dissolution products of BG to upregulate osteogenic gene expression in osteoblastic cells [36] or enhance local angiogenesis [23,37], and BG dissolves more rapidly than other bioceramics. Furthermore, the presence of different fillers may contribute to the degradation behavior of the ceramics and alter the local $\mathrm{pH}$ around the scaffold, hence contributing to the interaction of the material with the osteoblastic cells.

Biomaterials used for matrix construction possess distinct affinities for plasma proteins, which contribute to cellular adhesion and construct integration with surrounding bone [38]. Like many other synthetic polymers, PLG is hydrophobic, and the incorporation of bioceramic produced more hydrophilic scaffolds. As shown in earlier work from our laboratory and others [18], nanosized HA was uniformly incorporated into porous scaffolds using the gas foaming process, and we observed similar distribution for TCP and BG. Previous studies report greater hydrophilicity of HA versus TCP when measuring contact angles of water on homogeneous ceramics [39]. Trypan blue staining demonstrated that 
composite scaffolds have a substantial portion of exposed bioceramic from the polymer, which may provide binding sites for plasma proteins or cells when implanted or used as a cell delivery vehicle.

Osteoblasts play a critical role in the maintenance of mineral deposition and calcium-phosphate homeostasis. Bioceramics nucleate cell-secreted calcium and promote the formation of a mineralized microenvironment that directs subsequent osteoblast activity. Compared to osteoblasts on PLG control scaffolds, cells on HA- and TCP-PLG substrates exhibited lower expression of RUNX2 and COL1A1 at both time points. We have observed similar trends in osteogenic gene expression for human mesenchymal stem cells when cultured on PLG control substrates or PLG scaffolds coated with bone-like mineral, yet we observed increased ALP activity and calcium deposition [22]. This unexpected reduction may be due to a number of reasons. In the presence of increased concentrations of bone-like minerals, cells may alter their osteogenic program, thus shifting the temporal sequence of gene expression. Alternatively, proteins from the surrounding media may adsorb differentially to HA and TCP, thus initiating alternate integrin engagement and downstream signaling pathways that act alongside, or independent of $R U N X 2$ to modulate osteogenesis. In these studies, osteoblasts cultured on HA-PLG scaffolds demonstrated lower ALP activity and lower expression of RUNX2, COL1A1, and $S P A R C$, signifying a less potent osteogenic response compared to other materials. Conversely, osteoblasts cultured on BG-PLG scaffolds uniformly exhibited a greater osteogenic response after 21 days of culture. BG degrades much faster than HA and TCP, and bioactive glasses activate numerous cellular pathways including osteogenic differentiation, cellular proliferation and metabolism by stimulating neighboring cells with their ionic degradation products [33]. In addition, 45S5 ${ }^{\circledR}$ Bioglass contains silica, a constituent that is lacking from the HA and TCP employed in this study. In previous studies characterizing the response of human osteoblasts seeded on silica surfaces without the interference of other ions present in glass ceramics, there were no apparent differences in cell number, metabolic activity, or ALP activity, yet nodule formation was accelerated on silica surfaces [40]. The importance of silica is confirmed in other studies demonstrating significant increases in ALP activity and type I collagen production by osteoblasts exposed to bioactive glasses with $46.1 \mathrm{~mol} \%$ silica content (45S5) than cells exposed to bioactive glasses with 60 or $80 \mathrm{~mol} \%$ silica (58S and 77S, respectively) [41].

\section{Conclusions}

The results of this study demonstrate that bioceramic selection plays an important role in the resulting biophysical properties and osteogenic potential of $3 \mathrm{D}$ composite scaffolds for use in bone tissue engineering. Using a fabrication process that avoids excessive heat or harsh organic solvents, we produced macroporous, biodegradable composite materials using three widely used bioceramics with compressive moduli on the order of trabecular bone and possessing osteogenic potential. Furthermore, these data suggest that the physical properties and osteogenic response can be further tailored by increasing polymer-ceramic interactions or through incorporating other materials such as bioactive glasses with increased silica content. These observations and principles may be valuable to tailor the properties of the implant to specific bone defects or develop alternative in vitro models of bone formation. 


\section{Acknowledgments}

We thank Hillary Davis and Matt Mui for technical assistance with scanning electron microscopy. The authors are grateful to The Hartwell Foundation and the AO Research Fund of the AO Foundation (F-06-98L) for their generous financial support of this work (JKL). The project described was supported by Award Number T32EB003827 from the National Institute of Biomedical Imaging and Bioengineering (DGM). The content is solely the responsibility of the authors and does not necessarily represent the official views of the National Institute of Biomedical Imaging and Bioengineering or the National Institutes of Health. This work was also supported in part by the T32 Pre-Doctoral Clinical Research Training Program (UC Davis School of Medicine and Clinical and Translational Science Center).

\section{References}

1. Moroni, A.; Larsson, S.; Hoang Kim, A.; Gelsomini, L.; Giannoudis, P.V. Can we improve fixation and outcomes? Use of bone substitutes. J. Orthop. Trauma 2009, 23, 422-425.

2. El-Ghannam, A. Bone reconstruction: From bioceramics to tissue engineering. Expert Rev. Med. Devices 2005, 2, 87-101.

3. Loo, S.C.; Moore, T.; Banik, B.; Alexis, F. Biomedical applications of hydroxyapatite nanoparticles. Curr. Pharm. Biotechnol. 2010, 11, 333-342.

4. Wagoner Johnson, A.J.; Herschler, B.A. A review of the mechanical behavior of $\mathrm{CaP}$ and $\mathrm{CaP} /$ polymer composites for applications in bone replacement and repair. Acta Biomater. 2011, 7 , 16-30.

5. Rahaman, M.N.; Day, D.E.; Sonny Bal, B.; Fu, Q.; Jung, S.B.; Bonewald, L.F.; Tomsia, A.P. Bioactive glass in tissue engineering. Acta Biomater. 2011, 7, 2355-2373.

6. Gorustovich, A.A.; Roether, J.A.; Boccaccini, A.R. Effect of bioactive glasses on angiogenesis: A review of in vitro and in vivo evidences. Tissue Eng. Part B 2010, 16, 199-207.

7. Keating, J.F.; McQueen, M.M. Substitutes for autologous bone graft in orthopaedic trauma. J. Bone Joint Surg. Br. 2001, 83, 3-8.

8. Hutmacher, D.W. Scaffolds in tissue engineering bone and cartilage. Biomaterials 2000, 21, 2529-2543.

9. Tanaka, T.; Hirose, M.; Kotobuki, N.; Tadokoro, M.; Ohgushi, H.; Fukuchi, T.; Sato, J.; Seto, K. Bone augmentation by bone marrow mesenchymal stem cells cultured in three-dimensional biodegradable polymer scaffolds. J. Biomed. Mater. Res. A 2009, 91, 428-435.

10. Frohlich, M.; Grayson, W.L.; Wan, L.Q.; Marolt, D.; Drobnic, M.; Vunjak-Novakovic, G. Tissue engineered bone grafts: Biological requirements, tissue culture and clinical relevance. Curr. Stem Cell Res. Ther. 2008, 3, 254-264.

11. Ge, Z.; Tian, X.; Heng, B.C.; Fan, V.; Yeo, J.F.; Cao, T. Histological evaluation of osteogenesis of 3D-printed poly-lactic-co-glycolic acid (PLGA) scaffolds in a rabbit model. Biomed. Mater. 2009, 4, 021001:1-021001:7.

12. Davis, H.E.; Rao, R.R.; He, J.; Leach, J.K. Biomimetic scaffolds fabricated from apatite-coated polymer microspheres. J. Biomed. Mater. Res. A 2009, 90, 1021-1031. 
13. Lu, H.H.; El-Amin, S.F.; Scott, K.D.; Laurencin, C.T. Three-dimensional, bioactive, biodegradable, polymer-bioactive glass composite scaffolds with improved mechanical properties support collagen synthesis and mineralization of human osteoblast-like cells in vitro. J. Biomed. Mater. Res. A 2003, 64, 465-474.

14. Kim, S.S.; Ahn, K.M.; Park, M.S.; Lee, J.H.; Choi, C.Y.; Kim, B.S. A poly(lactide-coglycolide)/hydroxyapatite composite scaffold with enhanced osteoconductivity. J. Biomed. Mater. Res. A 2007, 80A, 206-215.

15. Daculsi, G.; Goyenvalle, E.; Cognet, R.; Aguado, E.; Suokas, E.O. Osteoconductive properties of poly(96L/4D-lactide)/beta-tricalcium phosphate in long term animal model. Biomaterials 2011, 32, 3166-3177.

16. Yao, J.; Radin, S.; Leboy, P.S.; Ducheyne, P. The effect of bioactive glass content on synthesis and bioactivity of composite poly (lactic-co-glycolic acid)/bioactive glass substrate for tissue engineering. Biomaterials 2005, 26, 1935-1943.

17. He, J.; Decaris, M.L.; Leach, J.K. Bioceramic-mediated trophic factor secretion by mesenchymal stem cells enhances in vitro endothelial cell persistence and in vivo angiogenesis. Tissue Eng. 2012, in press.

18. He, J.; Genetos, D.C.; Leach, J.K. Osteogenesis and trophic factor secretion are influenced by the composition of hydroxyapatite/poly(lactide-co-glycolide) composite scaffolds. Tissue Eng. Part A 2010, 16, 127-137.

19. Day, R.M.; Maquet, V.; Boccaccini, A.R.; Jerome, R.; Forbes, A. In vitro and in vivo analysis of macroporous biodegradable poly(D,L-lactide-co-glycolide) scaffolds containing bioactive glass. J. Biomed. Mater. Res. A 2005, 75, 778-787.

20. Leach, J.K.; Kaigler, D.; Wang, Z.; Krebsbach, P.H.; Mooney, D.J. Coating of VEGF-releasing scaffolds with bioactive glass for angiogenesis and bone regeneration. Biomaterials 2006, 27, 3249-3255.

21. Kim, S.S.; Sun Park, M.; Jeon, O.; Yong Choi, C.; Kim, B.S. Poly(lactide-coglycolide)/hydroxyapatite composite scaffolds for bone tissue engineering. Biomaterials 2006, 27, 1399-1409.

22. Davis, H.E.; Case, E.M.; Miller, S.L.; Genetos, D.C.; Leach, J.K. Osteogenic response to BMP-2 of hMSCs grown on apatite-coated scaffolds. Biotechnol. Bioeng. 2011, 108, 2727-2735.

23. Leu, A.; Leach, J.K. Proangiogenic potential of a collagen/bioactive glass substrate. Pharm. Res. 2008, 25, 1222-1229.

24. Schmittgen, T.D.; Livak, K.J. Analyzing real-time PCR data by the comparative C(T) method. Nat. Protoc. 2008, 3, 1101-1108.

25. Ge, C.; Xiao, G.; Jiang, D.; Yang, Q.; Hatch, N.E.; Roca, H.; Franceschi, R.T. Identification and functional characterization of ERK/MAPK phosphorylation sites in the Runx2 transcription factor. J. Biol. Chem. 2009, 284, 32533-32543.

26. Drissi, H.; Luc, Q.; Shakoori, R.; Chuva De Sousa Lopes, S.; Choi, J.Y.; Terry, A.; Hu, M.; Jones, S.; Neil, J.C.; Lian, J.B.; et al. Transcriptional autoregulation of the bone related CBFA1/RUNX2 gene. J. Cell. Physiol. 2000, 184, 341-350. 
27. Charles, P.; Mosekilde, L.; Risteli, L.; Risteli, J.; Eriksen, E.F. Assessment of bone remodeling using biochemical indicators of type I collagen synthesis and degradation: Relation to calcium kinetics. Bone Miner 1994, 24, 81-94.

28. Termine, J.D.; Kleinman, H.K.; Whitson, S.W.; Conn, K.M.; McGarvey, M.L.; Martin, G.R. Osteonectin, a bone-specific protein linking mineral to collagen. Cell 1981, 26, 99-105.

29. Carter, D.R.; Hayes, W.C. Bone compressive strength: The influence of density and strain rate. Science 1976, 194, 1174-1176.

30. Sicchieri, L.G.; Crippa, G.E.; de Oliveira, P.T.; Beloti, M.M.; Rosa, A.L. Pore size regulates cell and tissue interactions with PLGA-CaP scaffolds used for bone engineering. J. Tissue Eng. Regen. Med. 2012, 6, 155-162.

31. Rezwan, K.; Chen, Q.Z.; Blaker, J.J.; Boccaccini, A.R. Biodegradable and bioactive porous polymer/inorganic composite scaffolds for bone tissue engineering. Biomaterials 2006, 27 , 3413-3431.

32. Wei, G.; Ma, P.X. Structure and properties of nano-hydroxyapatite/polymer composite scaffolds for bone tissue engineering. Biomaterials 2004, 25, 4749-4757.

33. Hoppe, A.; Guldal, N.S.; Boccaccini, A.R. A review of the biological response to ionic dissolution products from bioactive glasses and glass-ceramics. Biomaterials 2011, 32, 2757-2774.

34. Scaglione, S.; Lazzarini, E.; Ilengo, C.; Quarto, R. A composite material model for improved bone formation. J. Tissue Eng. Regen. Med. 2010, 4, 505-513.

35. Smith, L.L.; Niziolek, P.J.; Haberstroh, K.M.; Nauman, E.A.; Webster, T.J. Decreased fibroblast and increased osteoblast adhesion on nanostructured NaOH-etched PLGA scaffolds. Int. J. Nanomedicine 2007, 2, 383-388.

36. Xynos, I.D.; Edgar, A.J.; Buttery, L.D.; Hench, L.L.; Polak, J.M. Gene-expression profiling of human osteoblasts following treatment with the ionic products of Bioglass 45S5 dissolution. J. Biomed. Mater. Res. 2001, 55, 151-157.

37. Leu, A.; Stieger, S.M.; Dayton, P.; Ferrara, K.W.; Leach, J.K. Angiogenic response to bioactive glass promotes bone healing in an irradiated calvarial defect. Tissue Eng. Part A 2009, 15, 877-885.

38. Webster, T.J.; Ergun, C.; Doremus, R.H.; Siegel, R.W.; Bizios, R. Specific proteins mediate enhanced osteoblast adhesion on nanophase ceramics. J. Biomed. Mater. Res. 2000, 51, 475-483.

39. Lopes, M.A.; Monteiro, F.J.; Santos, J.D.; Serro, A.P.; Saramago, B. Hydrophobicity, surface tension, and zeta potential measurements of glass-reinforced hydroxyapatite composites. J. Biomed. Mater. Res. 1999, 45, 370-375.

40. Anderson, S.I.; Downes, S.; Perry, C.C.; Caballero, A.M. Evaluation of the osteoblast response to a silica gel in vitro. J. Mater. Sci. Mater. Med. 1998, 9, 731-735.

41. Bosetti, M.; Zanardi, L.; Hench, L.; Cannas, M. Type I collagen production by osteoblast-like cells cultured in contact with different bioactive glasses. J. Biomed. Mater. Res. A 2003, 64, 189-195.

(C) 2012 by the authors; licensee MDPI, Basel, Switzerland. This article is an open access article distributed under the terms and conditions of the Creative Commons Attribution license (http://creativecommons.org/licenses/by/3.0/). 